ARTICLE

\title{
Proton-enabled activation of peptide materials for biological bimodal memory
}

Min-Kyu Song ${ }^{1}$, Seok Daniel Namgung (10 2 , Daehwan Choi ${ }^{1}$, Hyeohn Kim², Hongmin Seo ${ }^{2}$, Misong Ju², Yoon Ho Lee ${ }^{2}$, Taehoon Sung ${ }^{1}$, Yoon-Sik Lee ${ }^{3}$, Ki Tae Nam (i) ${ }^{2 凶} \&$ Jang-Yeon Kwon (1) ${ }^{1 凶}$

The process of memory and learning in biological systems is multimodal, as several kinds of input signals cooperatively determine the weight of information transfer and storage. This study describes a peptide-based platform of materials and devices that can control the coupled conduction of protons and electrons and thus create distinct regions of synapse-like performance depending on the proton activity. We utilized tyrosine-rich peptide-based films and generalized our principles by demonstrating both memristor and synaptic devices. Interestingly, even memristive behavior can be controlled by both voltage and humidity inputs, learning and forgetting process in the device can be initiated and terminated by protons alone in peptide films. We believe that this work can help to understand the mechanism of biological memory and lay a foundation to realize a brain-like device based on ions and electrons.

\footnotetext{
${ }^{1}$ School of Integrated Technology, Yonsei University, Incheon 21983, Republic of Korea. ${ }^{2}$ Department of Materials Science and Engineering, Seoul National University, Seoul 08826, Republic of Korea. ${ }^{3}$ School of Chemical and Biological Engineering, Nano Systems Institute, Seoul National University, Seoul 08826, Republic of Korea. ${ }^{凶}$ email: nkitae@snu.ac.kr; jangyeon@yonsei.ac.kr
} 
T he cooperative and multimodal activation process, which includes the transfer of ions or small molecules, specific recognition by receptors and subsequent cascade reactions by at least two inputs, is one of the most important characteristics of biological memory. For example, in a neuronal synapse, many chemicals such as neurotransmitters (e.g., dopamine), calcium ions, and protons cooperate in the process of electrical signal transfer, which, interestingly, depends on the external factors determined by sensory input or the memory of the previous events ${ }^{1,2}$. However, there is a large gap in our understanding and in controllability between electron-based synthetic devices and ion-based synaptic operation. Despite the advantage of the ability to harness coupled behaviors of ions and electrons as inputs, there are few reports of the application of such concerted behaviors to brain-inspired devices.

The proton-coupled mechanism that we focus on is ubiquitous in biological systems, as represented by proton-coupled electron transfer (PCET) $)^{3,4}$. Moreover, the role of protons in the synapse as neurotransmitters and mediators in cooperation with other neurotransmitters has been investigated in the past ${ }^{5,6}$. Proton-mediated signaling, in conjunction with conventional neurotransmitters, has been found mostly in sensory neurons in conditioned learning. Transient changes in extracellular proton concentrations are important in the activation of further signaling ${ }^{7,8}$. In this regard, along with the dopamine-based reward mechanism, proton-based processes can be applied to novel algorithms or synaptic devices. Our approach is based on one of the lessons learned from recent biological discoveries ${ }^{3-8}$ : that protons can facilitate metal redox and accumulate locally in electrically insulating peptide films.

The advantages of peptide materials are their structural programmability and versatile functionality, which can be controlled at a sequence level. Sequences can control folding, assembly, and other properties including inorganic material growth and electronic/ionic conductivities ${ }^{9-12}$. In this regard, peptides have been utilized even for nonbiomedical applications, including transistors, light-emitting devices, solar cells, batteries, and piezoelectric devices ${ }^{13-16}$. Previously, we identified the Tyr-Tyr-Ala-Cys-laTyr-Tyr (YYACAYY, Y7C) peptide, which can form a helix dimer and assemble spontaneously into a $2-\mathrm{D}$ film ${ }^{17}$. We have investigated the proton conductivity in manganese oxide hybridized Y7C films ${ }^{18}$. Through the measurement of the Onsager coefficient to understand the coupling of electrons and protons, it became evident that tyrosine can be involved in PCET and metal ion redox even in the thick films ${ }^{19}$. Thus, we explored the possibility of using the Y7C peptide for proton-mediated memory and synaptic devices.

Here, we demonstrate a programmable approach to protonregulated control based on tyrosine-rich peptide (TRP), providing a platform for designing and evaluating a bioinspired process of memory and learning algorithms. In this study, TRP is assembled into a film, and proton-dependent plasticity in conduction is utilized to activate memory and synaptic performance. We are further able to control the proton-controlled memory process and the timescale of learning and forgetting, which have never been realized with other materials.

\section{Results}

Film formation and electrochemical characterization. The Y7C peptide can exist as a dimer by disulfide bonding, exhibiting a helical structure, as confirmed by NMR (Fig. 1a, b). Because of the structural stability, this peptide can easily be assembled and deposited as a thick film by the spin-coating process (Fig. 1c). Circular dichroism analysis showed that the Y7C film had a helical conformation despite being made by spin-coating followed by a quick-drying process (Fig. 1d). Note that TRP exhibits different optical rotatory properties from typical helical peptides because of the absorbance of tyrosine groups ${ }^{17,20,21}$. The strong positive peak at $205 \mathrm{~nm}$ can be assigned to the amide $\pi-\pi^{*}$ transition and the $\pi-\pi^{*}$ transition of the phenolic side chain in an a-helical conformation at approximately $200 \mathrm{~nm}^{20}$. In addition, the positive peak at $232 \mathrm{~nm}$ found in the Y7C film corresponds to another $\pi-\pi^{*}$ transition of the phenolic side chain at $230 \mathrm{~nm}$ previously reported for $\mathrm{Y} 7 \mathrm{C}$ peptide in aqueous solution with an $\alpha$-helical conformation ${ }^{17}$.

The proton conduction property of the Y7C peptide was investigated using electrochemical impedance analysis (EIS). Ionblocking gold electrodes were utilized to measure capacitive effects solely from protons. Alternating current (AC) was applied with frequencies ranging from $20 \mathrm{~Hz}$ to $10^{6} \mathrm{~Hz}$ to obtain complex impedance plots with real parts on the $\mathrm{x}$-axis and imaginary parts on the y-axis, known as Nyquist plots (Fig. 1e). Proton conductivity was estimated by fitting the Nyquist plot to a typical resistor-capacitor (RC) circuit used for modeling proton exchange membranes (Supplementary Fig. 1 and Supplementary Note 1$)^{22-24}$. The Y7C film showed an increase in conductivity as the relative humidity (RH) increased (Fig. If and Supplementary Fig. 2). At $90 \% \mathrm{RH}$, the proton conductivity of the Y7C peptide reached $1.76 \times 10^{-2} \mathrm{~S} \mathrm{~cm}^{-1}$, which is comparable to the values of other proton conducting materials and biomaterials (Supplementary Table 1). Y7C showed approximately 20 times higher proton conductivity than FFACAFF, which contains phenylalanine instead of tyrosine, suggesting a role of the phenolic hydroxyl group in proton conduction. This value is higher than that of other tyrosine-containing peptides, such as YYDCDYY and YYCYY (Supplementary Fig. 3).

Proton-mediated resistive switching characteristics. Another interesting feature resulting from the high proton conductivity and redox capability of tyrosine is that the reduction and movement of silver ions inside the peptide film can be facilitated by both protons and applied electric fields (Supplementary Fig. 4). As a result, we discovered the proton-mediated resistive switching mechanism of Y7C film in a sandwiched structure between two electrodes (Fig. 2a and Methods). Highly insulating Y7C film in the pristine state becomes electrically conducting with the help of protons. When a positive voltage is applied, metal ions at the top electrode are generated and migrate to the bottom electrode, forming conducting filaments in the Y7C film via a mechanism similar to that of other resistive switching devices ${ }^{25-33}$. (Supplementary Note 2) However, a key difference is that under voltage bias, a phenolic hydroxyl group of tyrosine donates an electron to reduce Ag ions by generating a tyrosyl radical with the loss of one proton $^{34}$. The resulting $\mathrm{Ag}$ atom transfers an electron to the neighboring tyrosyl radical, forming tyrosine. The coupled redox of $\mathrm{Ag}$ ions and tyrosine results in the migration of $\mathrm{Ag}$ atoms, the formation of $\mathrm{Ag}$ clusters, and alignment into $\mathrm{Ag}$ filaments. Therefore, a sufficient level of positively biased voltage, defined as a set voltage $\left(\mathrm{V}_{\text {set }}=2.6 \mathrm{~V}\right.$ at $\left.45 \% \mathrm{RH}\right)$, induces an abrupt and dramatic increase of more than six orders of magnitude in the current. The transition from a high-resistance state (HRS) to a low-resistance state (LRS) is clearly observed. A detailed illustration of the proton-mediated resistive switching mechanism is provided in Supplementary Note 3.

The involvement of silver ions was confirmed by the finding that replacing the silver electrode with gold completely nullified the memristor performance (Supplementary Fig. 5). To understand the role of tyrosine in the resistance transition of peptides, the FFACAFF peptide was introduced. The set voltage of the FFACAFF film was increased to $10.7 \mathrm{~V}$ compared with $1.8 \mathrm{~V}$ for the Y7C film (Fig. 2b and Supplementary Fig. 6). This verifies that 
a

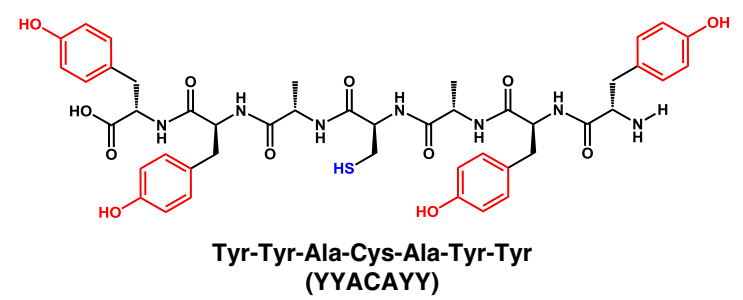

b

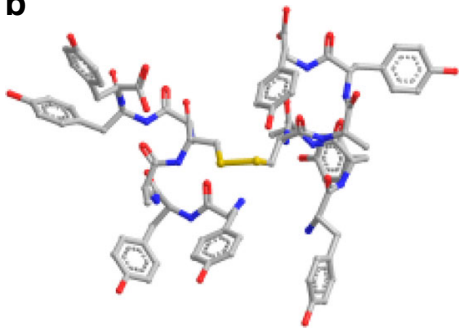

C

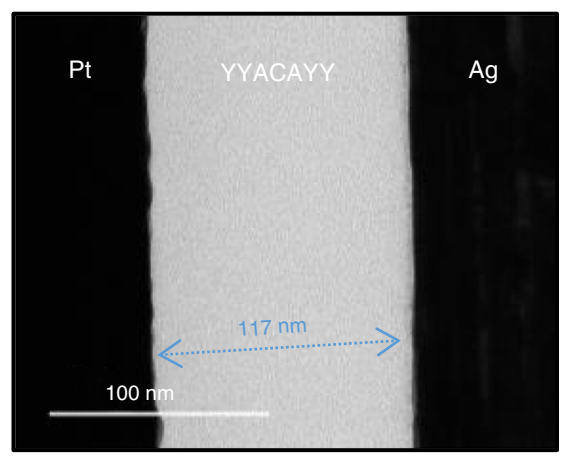

e

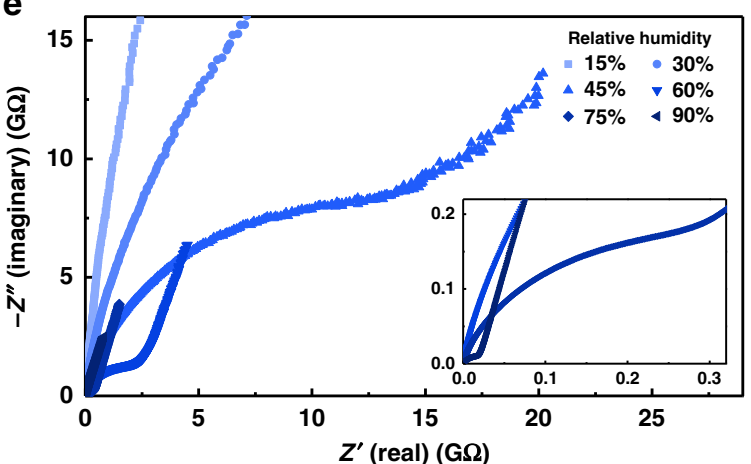

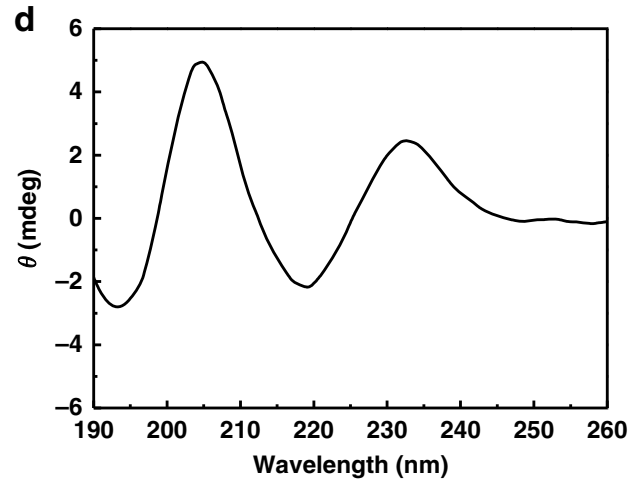

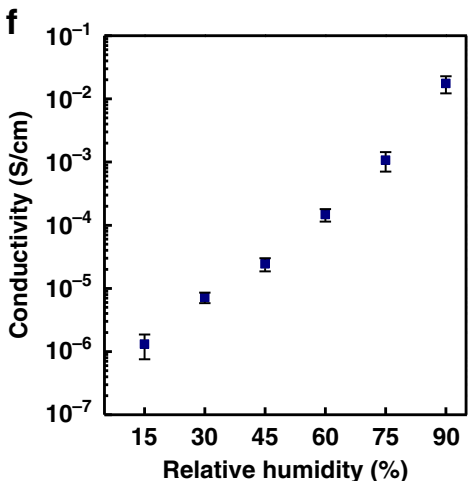

Fig. 1 Film formation and proton conduction of the Y7C peptide. (a) Chemical structure of the $Y 7 C$ peptide. The phenolic groups of tyrosine and thiol groups of cysteine are highlighted in red and blue, respectively. (b) Molecular structure of the dimer of Y7C revealed by NMR. Carbon, oxygen, and nitrogen are shown in gray, red, and purple, respectively. (c) Cross-sectional HRTEM image of the $Y 7 C$ film between Pt and Ag electrodes. A uniform and homogeneous film with a thickness of $117 \mathrm{~nm}$ can be identified between the Pt bottom electrode and the Ag top electrode. (d) Circular dichroism (CD) analysis of the $Y 7 C$ film. The solid CD spectrum is expressed in degrees of ellipticity ( $\theta$ ) without concentration terms. (e) Nyquist plots measured from the Y7C film in contact with two Au electrodes at different RH. The inset shows the magnified range at RH values of $60 \%, 75 \%$, and $90 \%$. (f) Conductivity of the $Y 7 C$ film calculated from the Nyquist plot as a function of RH. Error bars represent the mean \pm SD.

the hydroxyl group in tyrosine reduces the energy barrier to the reduction of $\mathrm{Ag}$ ions and promotes the formation of a conduction path (Supplementary Fig. 7). These results indicate that the resistive switching characteristics of peptide films can be controlled by specific peptide sequences.

We examined the direct correlation between the resistive switching characteristic and the proton conduction of the peptide. The $I-V$ characteristics of the peptide film under various $\mathrm{RH}$ conditions were obtained to verify the proton conduction effects by which the switching characteristic is modulated (Fig. 2c and Supplementary Note 4). Increasing RH induces a higher initial current at HRS by virtue of higher proton conduction. Moreover, the set voltage of the Y7C film decreased by more than one order of magnitude from $4.6 \mathrm{~V}$ to $0.4 \mathrm{~V}$ when $\mathrm{RH}$ increased from $15 \%$ to $90 \%$. The changes in set voltage depending on various $\mathrm{RH}$ conditions are summarized in Fig. 2e. The switching endurance, temporal variation, spatial variation, and data retention under various $\mathrm{RH}$ conditions are summarized in Supplementary Fig. 8.
The kinetic isotope effect (KIE) was studied with deuterium oxide $\left(\mathrm{D}_{2} \mathrm{O}\right)$ vapor instead of $\mathrm{H}_{2} \mathrm{O}$ to determine direct evidence of the role of protons in facilitating resistive switching. Even with $\mathrm{D}_{2} \mathrm{O}$ vapor, resistive switching behaviors can also be shown. Showing a similar tendency, the set voltages decrease with increasing $\mathrm{RH}$ from $5.6 \mathrm{~V}$ at $15 \%$ to $2.1 \mathrm{~V}$ at $90 \%$ (Fig. $2 \mathrm{~d}$ and Supplementary Fig. 9). However, the value of the set voltage with $\mathrm{D}_{2} \mathrm{O}$ is higher than that with $\mathrm{H}_{2} \mathrm{O}$. For example, at $\mathrm{RH} 90 \%$, the set voltage is $2.1 \mathrm{~V}$ with $\mathrm{D}_{2} \mathrm{O}$ but $0.4 \mathrm{~V}$ with $\mathrm{H}_{2} \mathrm{O}$. Because the set voltage directly reflects the kinetic energy barrier, the higher value means that more energy is required for the heavier deuterium in the series of concerted processes involved in silver redox and ionic conduction. Therefore, this set of experiments led to the conclusion that protons are directly involved in the resistive switching mechanism. Based on this understanding of protondependent resistive switching characteristics, we demonstrate a memristor controlled bimodally and independently by either electron or proton inputs and simultaneously by both. To the best of our knowledge, there is no prior memory device operated only 
a

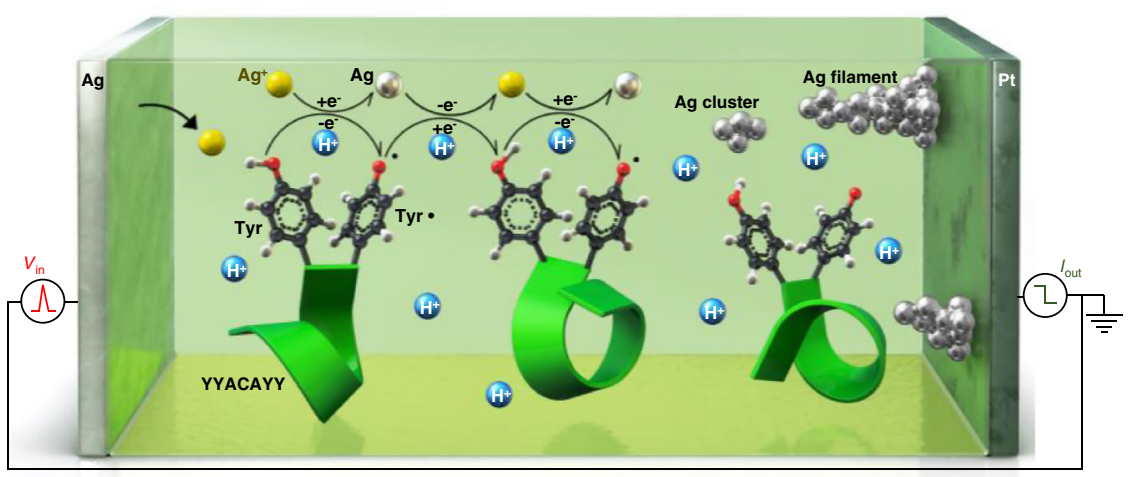

C

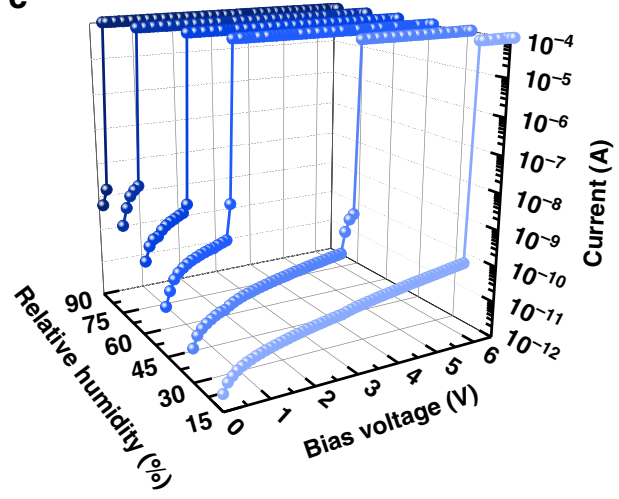

d

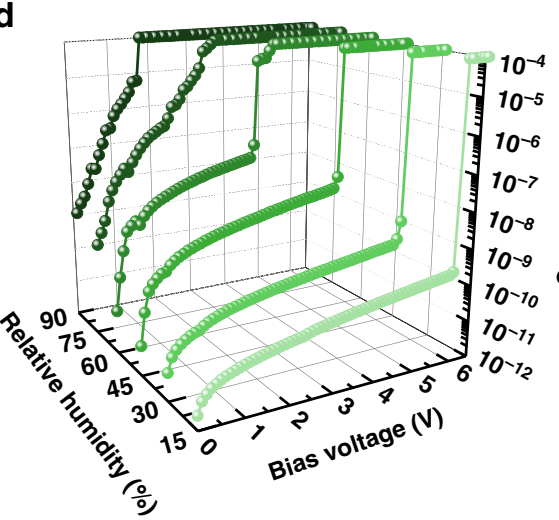

b

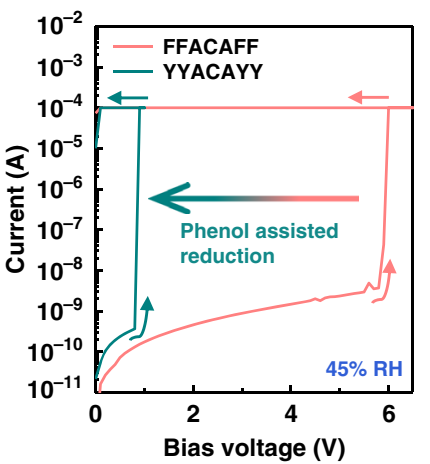

e

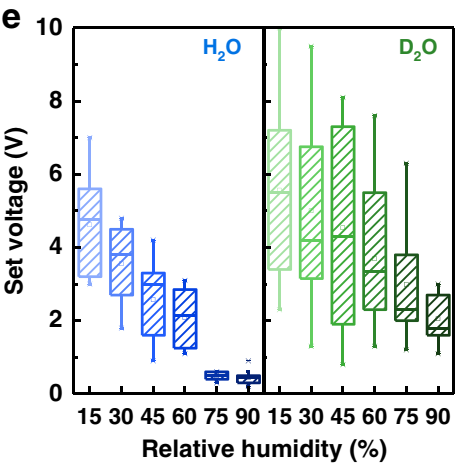

Fig. 2 Proton-mediated resistive switching characteristics of the Y7C peptide. (a) Schematic diagram of proton-mediated resistive switching of the $Y 7 C$ film. Helical peptide backbones are visualized as green ribbons. (b) Resistive switching characteristics of the Y7C peptide (emerald) and FFACAFF peptide (pink). Insets show the chemical structures of tyrosine and phenylalanine. (c, d) Resistive switching characteristics of $Y 7 C$ under various relative humidities of $\mathrm{H}_{2} \mathrm{O}(\mathbf{c})$ and $\mathrm{D}_{2} \mathrm{O}$ (d) vapor. Increasing humidity is expressed in increasing darkness of colors. Each curve was measured after $2 \mathrm{~h}$ of fixed RH conditions.

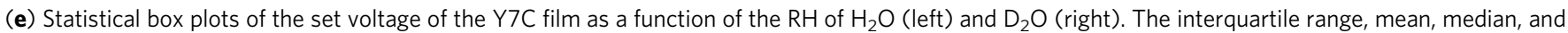
outliers are represented by boxes, open squares, central lines, and whiskers, respectively.

by ion-based stimuli such as calcium ions, sodium ions, or protons.

Proton/electron controlled bimodal memristor. The Y7C peptide film exhibits excellent resistive switching characteristics similar to other conventional memristors in with clear set and reset phenomena and a reasonable on/off current ratio of $10^{6}$ at $45 \%$ RH (Fig. 3a and Supplementary Fig. 10) ${ }^{35-37}$. Surprisingly, the resistive switching characteristics of the Y7C film were controlled only by RH change instead of electrical bias (Fig. 3b). $\mathrm{RH}$ was controlled by the injection of either $\mathrm{N}_{2}$ gas to reduce humidity or $\mathrm{H}_{2} \mathrm{O}$ humidified air to increase humidity and detected simultaneously by a humidity sensor (Supplementary Fig. 11 and Supplementary Note 5). With a constant read voltage of $0.3 \mathrm{~V}$, a forward RH sweep from $5 \%$ to $95 \%$ was applied. The current abruptly increased by more than three orders of magnitude when RH reached $\sim 90 \%$, which corresponds to clear set operation to LRS $\left(\mathrm{RH}_{\mathrm{set}}\right)$. When a subsequent reverse $\mathrm{RH}$ sweep from $95 \%$ to $5 \%$ was applied, the current rapidly decreased at an $\mathrm{RH}$ of $\sim 5 \%$, corresponding to a clear reset operation to HRS $\left(\mathrm{RH}_{\text {reset }}\right)$ (Supplementary Fig. 12 and Supplementary Note 6). Resistive switching of the Y7C film with an on/off ratio over $10^{4}$ is obviously controlled by $\mathrm{RH}$ alone. As the variation in RH leads to the change in proton conduction, resistive switching of the device is controlled by protons. The device retains its data states for over $10^{4} \mathrm{~s}$ regardless of whether it is written by voltage bias or by humidity (Fig. 3c).

As the peptide memristor operated by two individual modes, bias mode and humidity mode, was examined, the complementary bimodal operation of the peptide memristor was carried out (Fig. 3d). Because Ag redox for the Ag filament formation is coupled by the proton concentration and the switching by humidity is induced from the lowered onset potential, the LRS set by the humidity at low voltage bias can be erased by the electrical pulse. The data state of the peptide memristor was written by applying a positive voltage bias of $5 \mathrm{~V}$ and erased by a negative voltage bias of $-5 \mathrm{~V}$. Subsequently, the set operation was driven by increasing $\mathrm{RH}$ to $90 \%$, and then the reset operation was successfully conducted by a negative voltage bias. Dual inputs using voltage and humidity, which correspond to electrons and protons, respectively, can complementarily write and erase data in one device (Supplementary Note 6 and 7).

Proton-activated artificial synapses. The concept of protonactivated devices can be further extended to short-term memory, synaptic transistors. Synaptic plasticity in terms of the ability of synapses to strengthen connectivity over time indicates that the learning process in the brain is not a unidirectional single-variable function from presynaptic inputs to postsynaptic current. It is a multivariable function in which the degree of learning is manipulated by interactions between a signaling agent and its environment. In particular, it has been proven that protons play modulatory roles that potentiate and/or inhibit neuronal ion channels ${ }^{8}$. Acid-sensing ion channels (ASICs), which detect extracellular proton concentration, function as signal receptors for proton signaling systems. In addition, the activities of other ion channels, including calcium, sodium, and potassium channels and glutamate and GABA receptors, are modulated by extracellular 

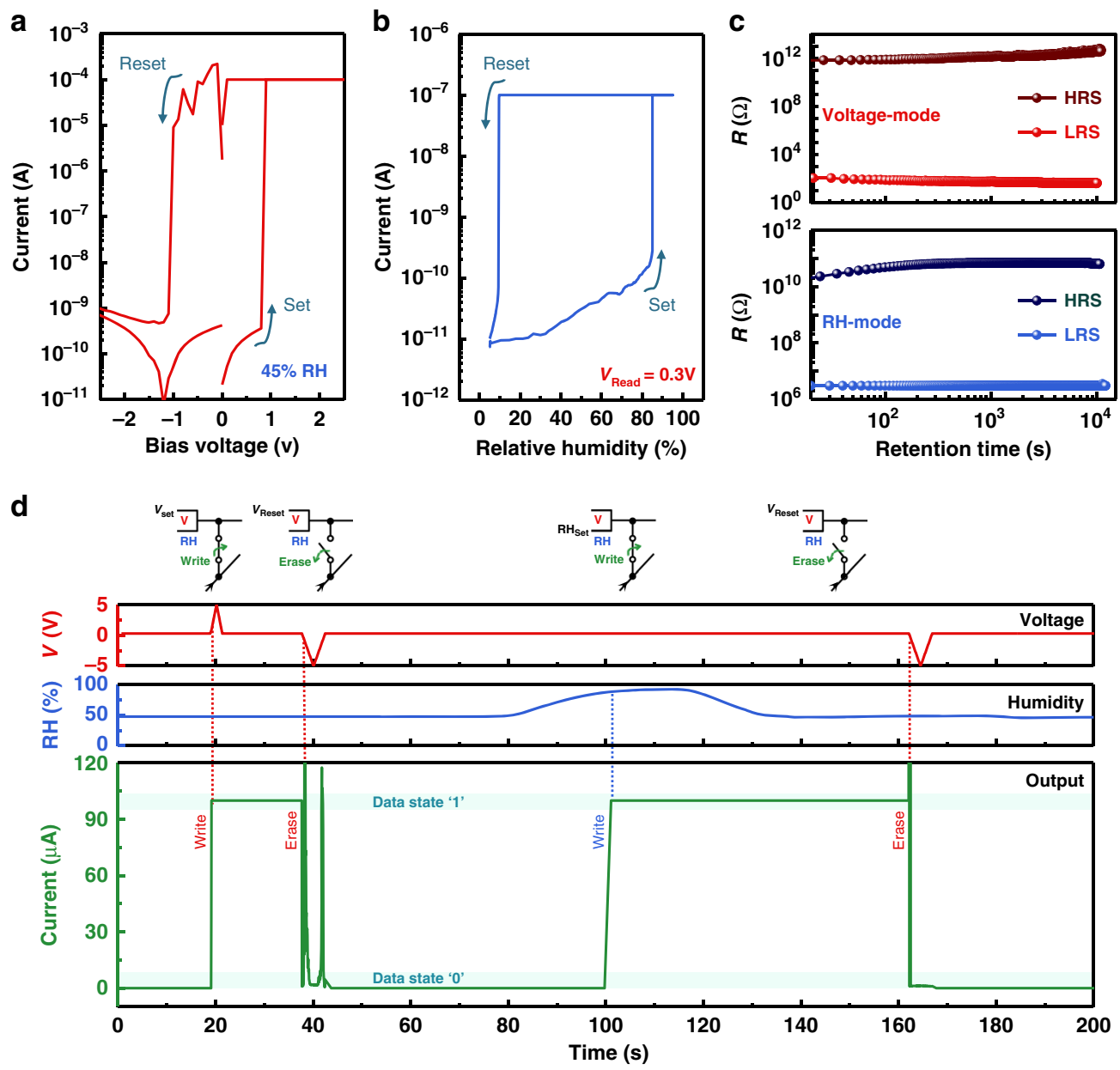

Fig. 3 Proton/electron controlled bimodal memristor based on the Y7C peptide. (a) Current-voltage characteristics of the device at a relative humidity of $45 \%$. The voltage sweep direction $(0 \rightarrow 2 \rightarrow-2 \rightarrow 0 \mathrm{~V})$ is displayed with navy arrows. (b) Current humidity characteristics of the device with a constant read voltage of $0.3 \mathrm{~V}$. The $\mathrm{RH}$ sweep direction $(5 \rightarrow 95 \rightarrow 5 \%)$ is displayed with navy arrows. (c) Data retention characteristics of the device in voltage mode (top) and RH mode (bottom). (d) The current variation of the $\mathrm{Y} 7 \mathrm{C}$ peptide memristor controlled by voltage and/or RH input, clearly showing bimodal operation. Voltage pulse, RH pulse, and current output are represented by red, blue and green curves, respectively.

proton concentration. For instance, GluK2/K4 kainate receptors are potentiated by protons (Fig. $4 \mathrm{a}$, top) ${ }^{38}$. In this regard, we expect that the peptide enables the proton activation process and regulation of the synaptic response.

For the fabrication of proton-controlled synaptic transistors, Y7C, In-Ga-Zn-O (IGZO), and $\mathrm{Au}$ were used for the gate dielectric, semiconductor, and metal, respectively (Fig. 4a, bottom and Methods). The Y7C film was spin-coated prior to the deposition of the channel and electrode layers for the in-planegate configuration. A lateral gate and IGZO channel were used for presynaptic terminal and postsynaptic neurons, respectively. The IGZO is used as the channel material due to its high on/off ratio, low leakage current, and process compatibility to the Y7C peptide film (Supplementary Note 8). Mobile protons in the Y7C electrolyte film play a role as the neurotransmitter ${ }^{39}$. The synaptic plasticity of the synaptic device was investigated by applying a presynaptic input voltage to the gate and measuring the excitatory postsynaptic current (EPSC) at the source/drain. Mimicking the proton-controlled regulation of biological neurotransmission, the synaptic response of the devices is activated under the protonic stimulus, and the plasticity can be precisely controlled. At low $\mathrm{RH}$ (under $80 \%$ ), there was no drain current change when two consecutive triangular-shaped voltage pulses were applied to the gate (Fig. 4b). However, at RH values over $90 \%$, which is sufficient for proton modulation, the synaptic response was activated due to the accumulation and depletion of protons in the hydrated peptide film (Supplementary Figs. 13 and 14). The drain current to a single pulse exhibited a typical EPSC response of biological synapses, showing a sudden increase and gradual decay depending on the spike duration (Supplementary Fig. 15a). When paired pulses were applied, the EPSC response to the second pulse was potentiated by $110 \%$ compared to the first response, representing paired-pulse facilitation (PPF) (Fig. 4b). The PPF index, defined as the ratio of the second peak to the first peak, is a function of the time interval between two pulses. The maximum PPF index was $151 \%$ with $\Delta \mathrm{t}=100 \mathrm{~ms}$ (Supplementary Fig. 15b). Data retention after stimulation, which is closely related to memory ability, was strengthened depending on the amplitude of stimulations, corresponding to the transition from short-term plasticity (STP) to long-term plasticity (LTP) of the biological synapse (Supplementary Fig. 15c). When the amplitude of the presynaptic voltage was in the range between $0.1 \mathrm{~V}$ and $2 \mathrm{~V}$, the relaxation time was relatively short term within a few seconds. On the other hand, when the amplitude of the presynaptic voltage was larger than $2 \mathrm{~V}$, the relaxation time increased to a few hundred seconds. (Supplementary Fig. 15d) In addition, spikenumber dependent plasticity (SNDP) and spike-frequency dependent plasticity (SFDP) were observed by varying the stimulation number and the pulse frequency, respectively. When the number of stimulations increased from 2 to 20, the relaxation 
a

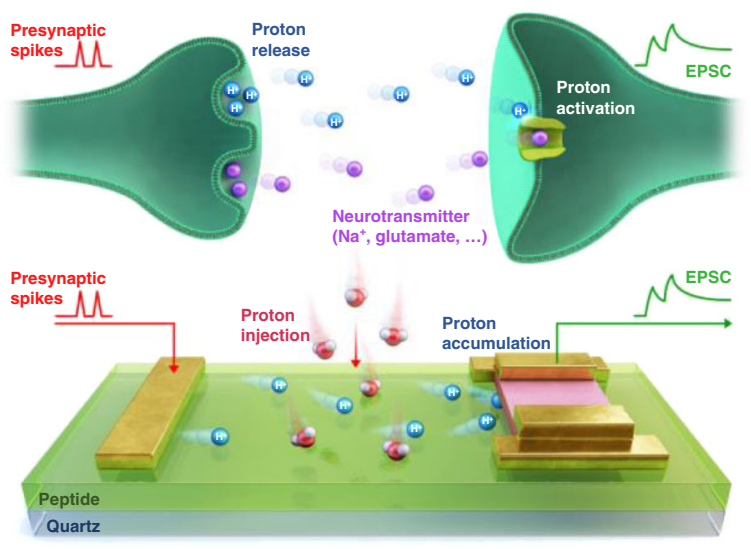

b

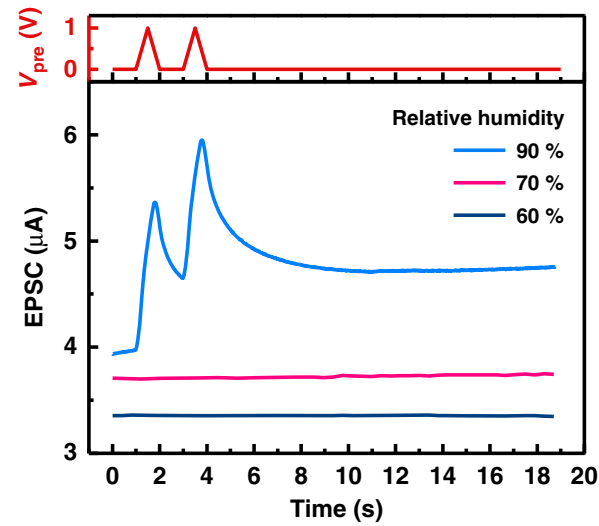

c

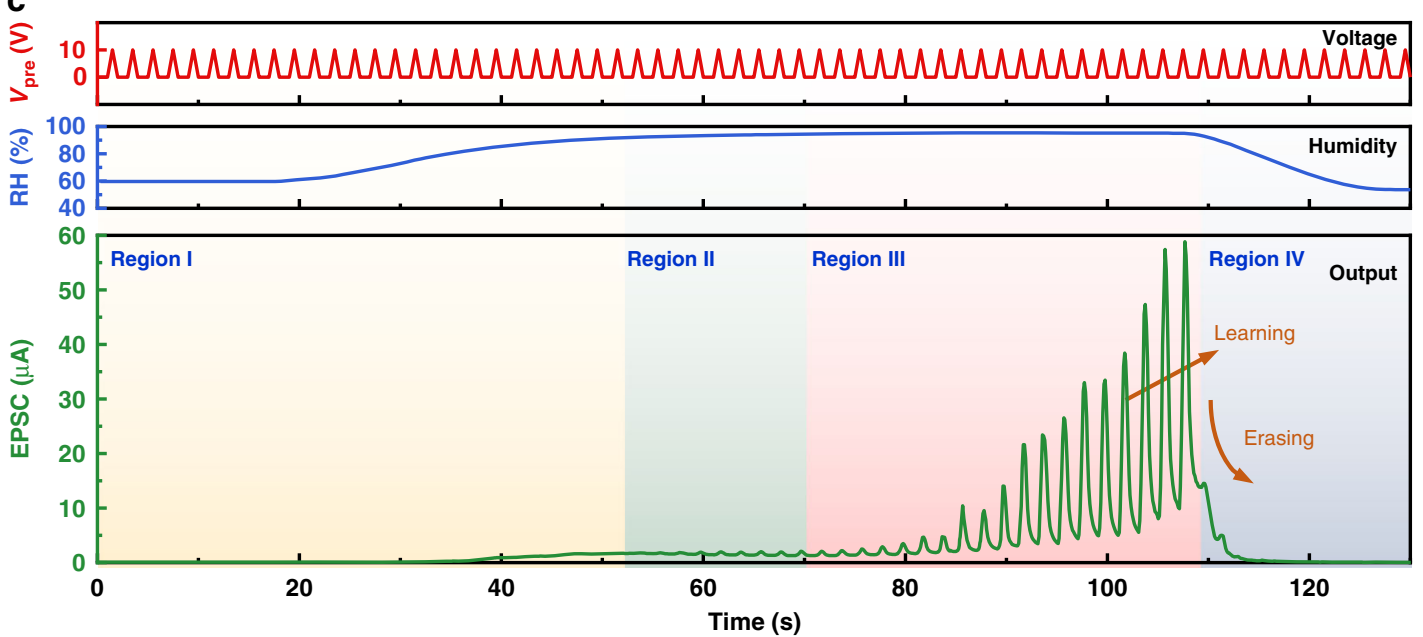

Fig. 4 Proton-activated artificial synapses based on the Y7C peptide. (a) Schematics of the proton activation process in biological synapses (top) and artificial synapses (bottom). Presynaptic spikes and corresponding EPSCs are shown in red and green, respectively. In biological synapses, protons (blue) released from presynaptic neurons activate receptors at postsynaptic neurons to receive neurotransmitters (purple). (b) Postsynaptic currents of the artificial synapse under various $\mathrm{RH}$ conditions triggered by a pair of presynaptic voltage pulses $(\mathrm{V}=1 \mathrm{v}, \Delta \mathrm{t}=1 \mathrm{~s})$. PPF occurred at $90 \% \mathrm{RH}$ (blue), while there was no presynaptic response at either $70 \% \mathrm{RH}$ (magenta) or $60 \% \mathrm{RH}$ (navy). (c) Proton-dependent plasticity of artificial synapses. The voltage pulse, $\mathrm{RH}$ pulse, and corresponding current output of the device are shown as red, blue, and green curves, respectively. Only when both voltage and RH inputs are activated does a notable learning process appear, and the synaptic response is suppressed drastically by reducing $\mathrm{RH}$.

time of the data increased (Supplementary Fig. 15e). EPSC potentiation triggered by 10 pulses was enhanced by amounts ranging from $110 \%$ for the $1 \mathrm{~Hz}$ case to $2120 \%$ for the $10 \mathrm{~Hz}$ case (Supplementary Fig. 15f). The spatial and temporal variations of the activated artificial synapse are summarized in Supplementary Fig. 16 and Supplementary Note 9.

The time-scaled electrical characteristics of proton-dependent synaptic transistors were studied (Fig. 4c). Electrical presynaptic inputs with a pulse width of $1 \mathrm{~s}$ were sequentially applied while the modulatory $\mathrm{RH}$ input was varied. The proton-dependent plasticity in the postsynaptic current can be divided into 4 regions. In region I at low RH, EPSC was not affected by voltage input. When RH was increasing, EPSC started to increase because proton conduction increases with RH. In region II, the postsynaptic current started to respond to voltage inputs when $\mathrm{RH}$ exceeded $90 \%$, while no sequential pulse facilitation was observed. Over time, a remarkable learning process appeared starting at $70 \mathrm{~s}$ at $\mathrm{RH}$ above $94 \%$ (in region III). In this high- $\mathrm{RH}$ region, the postsynaptic current was continuously potentiated as a presynaptic voltage pulse train was applied. When $\mathrm{RH}$ was decreased in region IV, the synaptic response was suppressed immediately, indicating that proton-induced synaptic connectivity was inhibited. This proton-activated synaptic plasticity occurred because proton accumulation and depletion at the interface of the Y7C film/IGZO took place as $\mathrm{RH}$ reached a threshold value of $94 \%$. Accumulated protons resulted in the formation of an electric double layer (EDL), which showed a large capacitance and long-range lateral electrostatic coupling, in a similar mechanism as observed in other synaptic devices ${ }^{40-45}$. The slow relaxation resulting from low ionic mobility can induce learning and forgetting phenomena ${ }^{46-49}$. We further demonstrated the controllability of capacitive effects from proton accumulation by $\mathrm{RH}$ control so that the synaptic response could be activated and deactivated. The proton-sensitive feature of the Y7C peptide enables proton as a modulatory input to regulate whether to operate in the proton-gated transistors (Supplementary Note 10). Furthermore, our results are analogous to the multivariable functions of proton-sensitive neurotransmission, where synaptic learning behavior is affected by extracellular proton concentration.

\section{Discussion}

In summary, our study demonstrates unprecedented multimodal neuron mimetic operations based on the control of proton activity in redox-active TRP films. The strategy developed here 
may embody new recent findings associated with biological reward-based learning, where the strength of synaptic connections is modulated. Furthermore, our observation emphasizes the importance of proton utilization in the reinforcing and switching of devices. We expect that this TRP film-based platform can be extended to other ions- and/or small molecule-based artificial sensory synapses on multiple timescales.

\section{Methods}

Circular dichroism analysis. For the $\mathrm{CD}$ analysis, a Y7C film was made via spincoating a $1 \mathrm{wt} \% \mathrm{Y} 7 \mathrm{C}$ solution onto a quartz substrate. The $\mathrm{CD}$ spectrum of the $\mathrm{Y} 7 \mathrm{C}$ film was recorded using a J-815 spectropolarimeter (Jasco) at $25^{\circ} \mathrm{C}$. The spectrum was collected from 260 to $180 \mathrm{~nm}$ with a $0.1 \mathrm{~nm}$ interval keeping the HT voltage less than $600 \mathrm{~V}$ for reliability. The data from 3 scans were averaged for each spectrum for reproducibility.

Impedance analysis. Impedance analysis of the peptide films was carried out in the frequency range of $20 \mathrm{~Hz}$ to $1 \mathrm{MHz}$ using an impedance analyzer (Keysight Technologies, E4990A). The real and imaginary impedance, magnitude, and phase angle were measured. An oscillator voltage of $500 \mathrm{mV}$ and a DC bias voltage of 100 $\mathrm{mV}$ was applied.

Fabrication of the memristor. A $10 \mathrm{~nm}$ Cr adhesion layer and a $100 \mathrm{~nm}$ Pt bottom electrode were first deposited on $\mathrm{SiO}_{2} / \mathrm{Si}$ substrate by direct current (DC) sputtering. A $2 \mathrm{wt} \%$ Y7C peptide solution was prepared by dissolving Y7C peptide powder (Beadtech, 95.08\%) in trifluoroacetic acid (Daejung, 99.0\%) at room temperature. The Y7C solution was spin-coated onto the substrates at $4000 \mathrm{rpm}$ for $60 \mathrm{~s}$, followed by $\mathrm{Ag}$ top electrode deposition by thermal evaporation at a pressure of $\approx 10^{-6}$ torr. The active area of $200 \times 200 \mu \mathrm{m}$ was defined by a shadow mask during the top electrode deposition. A homogeneous film with clear interfaces was confirmed by transmission electron microscopy (TEM) (Fig. 1c).

Fabrication of the synaptic transistor. A $4 \mathrm{wt} \%$ Y7C peptide solution was spincoated onto quartz substrates at $4000 \mathrm{rpm}$ for $60 \mathrm{~s}$. Then, 50-nm-thick In-Ga-Zn-O active layers were defined by a shadow mask and deposited by RF sputtering. The sputtering was performed at room temperature with a base pressure of $<10^{-6}$ torr, an RF power of $100 \mathrm{~W}$, and gas flow rates of $30 \mathrm{sccm}$ and $0.5 \mathrm{sccm}$ for $\mathrm{Ar}$ and $\mathrm{O}_{2}$, respectively. Then, $100-\mathrm{nm}$-thick $\mathrm{Au}$ with $10-\mathrm{nm}$-thick $\mathrm{Ni}$ as an adhesion layer defined by the shadow mask was thermally evaporated onto the channel layer (Fig. 4a).

\section{Data availability}

All data is available in the main text or the supplementary information.

Received: 27 May 2020; Accepted: 29 October 2020;

Published online: 19 November 2020

\section{References}

1. Neftci, E. O. \& Averbeck, B. B. Reinforcement learning in artificial and biological systems. Nat. Mach. Intell. 1, 133-143 (2019).

2. Sasaki, M., Takagi, M. \& Okamura, Y. A voltage sensor-domain protein is a voltage-gated proton channel. Science 312, 589-592 (2006).

3. Reichard, P. \& Ehrenberg, A. Ribonucleotide reductase-a radical enzyme Science 221, 514-519 (1983).

4. Meyer, T. J., Huynh, M. H. \& Thorp, H. H. The possible role of protoncoupled electron transfer (PCET) in water oxidation by photosystem II. Angew. Chem. Int. Ed. 46, 5284-5304 (2007).

5. Chesler, M. Regulation and modulation of $\mathrm{pH}$ in the brain. Physiol. Rev. 83, 1183-1221 (2003).

6. Beg, A. A., Ernstrom, G. G., Nix, P., Davis, M. W. \& Jorgensen, E. M. Protons act as a transmitter for muscle contraction in C. elegans. Cell 132, 149-160 (2008).

7. Tombaugh, G. C. \& Somjen, G. G. Effects of extracellular pH on voltage-gated $\mathrm{Na}^{+}, \mathrm{K}^{+}$and $\mathrm{Ca}^{2+}$ currents in isolated rat CA1 neurons. J. Physiol. 493, 719-732 (1996).

8. Wemmie, J. A., Taugher, R. J. \& Kreple, C. J. Acid-sensing ion channels in pain and disease. Nat. Rev. Neurosci. 14, 461-471 (2013).

9. Daura, X. et al. Peptide folding: When simulation meets experiment. Angew. Chem. Int. Ed. 38, 236-240 (1999).

10. Hartgerink, J. D., Beniash, E. \& Stupp, S. I. Self-assembly and mineralization of peptide-amphiphile nanofibers. Science 294, 1684-1688 (2001).
11. Amit, M., Yuran, S., Gazit, E., Reches, M. \& Ashkenasy, N. Tailor-made functional peptide self-assembling nanostructures. Adv. Mater. 30, 1707083 (2018).

12. Lee, H. E. et al. Amino-acid- and peptide-directed synthesis of chiral plasmonic gold nanoparticles. Nature 556, 360-365 (2018).

13. Berger, O. et al. Light-emitting self-assembled peptide nucleic acids exhibit both stacking interactions and Watson-Crick base pairing. Nat. Nanotechnol. 10, 353 (2015).

14. Tao, K., Makam, P., Aizen, R. \& Gazit, E. Self-assembling peptide semiconductors. Science 358, eaam9756 (2017).

15. Song, M.-K. et al. Physically transient field-effect transistors based on black phosphorus. ACS Appl. Mater. Inter. 10, 42630-42636 (2018).

16. Namgung, S. D. et al. Tyrosine-rich peptide insulator for rapidly dissolving transient electronics. Adv. Mater. Technol. 5, 2000516 (2020).

17. Jang, H. S. et al. Tyrosine-mediated two-dimensional peptide assembly and its role as a bio-inspired catalytic scaffold. Nat. Commun. 5, 1-11 (2014).

18. Lee, J. et al. Proton conduction in a tyrosine-rich peptide/manganese oxide hybrid nanofilm. Adv. Funct. Mater. 27, 1702185 (2017).

19. Ju, M. et al. Quantitative analysis of the coupling between proton and electron transport in peptide/manganese oxide hybrid films. Phys. Chem. Chem. Phys. 22, 7537-7545 (2020).

20. Chen, A. K. \& Woody, R. W. A theoretical study of the optical rotatory properties of poly-L-tyrosine. J. Am. Chem. Soc. 93, 29-37 (1971).

21. Engel, J., Liehl, E. \& Sorg, C. Circular dichroism, optical rotatory dispersion and helix $\rightleftharpoons$ coil transition of polytyrosine and tyrosine peptides in nonaqueous solvents. Eur. J. Biochem. 21, 22-30 (1971).

22. Garrido, L., Lopez-Gonzalez, M., Tlenkopatchev, M. \& Riande, E. Proton diffusion in polyelectrolytes based on hydrogenated polynorbornenes with imide side groups in the repeat unit as determined by NMR and impedance spectroscopies. J. Membr. Sci. 380, 199-207 (2011).

23. Ordinario, D. D. et al. Bulk protonic conductivity in a cephalopod structural protein. Nat. Chem. 6, 596-602 (2014).

24. Wünsche, J. et al. Protonic and electronic transport in hydrated thin films of the pigment eumelanin. Chem. Mater. 27, 436-442 (2015).

25. Tseng, R. J. et al. Digital memory device based on tobacco mosaic virus conjugated with nanoparticles. Nat. Nanotechnol. 1, 72-77 (2006).

26. Jo, S. H. et al. Nanoscale memristor device as synapse in neuromorphic systems. Nano Lett. 10, 1297-1301 (2010).

27. Wang, $H$. et al. Sericin for resistance switching device with multilevel nonvolatile memory. Adv. Mater. 25, 5498-5503 (2013).

28. Messerschmitt, F., Kubicek, M. \& Rupp, J. L. How does moisture affect the physical property of memristance for anionic-electronic resistive switching memories? Adv. Funct. Mater. 25, 5117-5125 (2015).

29. Di Mauro, E. et al. Resistive switching controlled by the hydration level in thin films of the biopigment eumelanin. J. Mater. Chem. C 4, 9544-9553 (2016).

30. Choi, S. et al. SiGe epitaxial memory for neuromorphic computing with reproducible high performance based on engineered dislocations. Nat. Mater. 17, 335-340 (2018).

31. Fuller, E. J. et al. Parallel programming of an ionic floating-gate memory array for scalable neuromorphic computing. Science 364, 570-574 (2019).

32. Xia, Q. \& Yang, J. J. Memristive crossbar arrays for brain-inspired computing. Nat. Mater. 18, 309-323 (2019).

33. Yeon, H. et al. Alloying conducting channels for reliable neuromorphic computing. Nat. Nanotechnol. 15, 574-579 (2020).

34. Sjödin, M. et al. Switching the redox mechanism: models for proton-coupled electron transfer from tyrosine and tryptophan. J. Am. Chem. Soc. 127, 3855-3863 (2005)

35. Strukov, D. B., Snider, G. S., Stewart, D. R. \& Williams, R. S. The missing memristor found. Nature 453, 80-83 (2008).

36. Hota, M. K., Bera, M. K., Kundu, B., Kundu, S. C. \& Maiti, C. K. A natural silk fibroin protein-based transparent bio-memristor. Adv. Funct. Mater. 22, 4493-4499 (2012).

37. Sangwan, V. K. et al. Multi-terminal memtransistors from polycrystalline monolayer molybdenum disulfide. Nature 554, 500-504 (2018).

38. Mott, D. D., Washburn, M. S., Zhang, S. \& Dingledine, R. J. Subunitdependent modulation of kainate receptors by extracellular protons and polyamines. J. Neurosci. 23, 1179-1188 (2003).

39. $\mathrm{Du}, \mathrm{J}$. et al. Protons are a neurotransmitter that regulates synaptic plasticity in the lateral amygdala. Proc. Natl Acad. Sci. USA 111, 8961-8966 (2014).

40. Zhu, L. Q., Wan, C. J., Guo, L. Q., Shi, Y. \& Wan, Q. Artificial synapse network on inorganic proton conductor for neuromorphic systems. Nat. Commun. 5, 3158 (2014).

41. van de Burgt, Y. et al. A non-volatile organic electrochemical device as a lowvoltage artificial synapse for neuromorphic computing. Nat. Mater. 16, 414-418 (2017).

42. Yang, C. S. et al. A synaptic transistor based on quasi-2D molybdenum oxide. Adv. Mater. 29, 1700906 (2017) 
43. Kim, Y. et al. A bioinspired flexible organic artificial afferent nerve. Science 360, 998-1003 (2018).

44. Seo, S. et al. Artificial optic-neural synapse for colored and color-mixed pattern recognition. Nat. Commun. 9, 5106 (2018).

45. van De Burgt, Y., Melianas, A., Keene, S. T., Malliaras, G. \& Salleo, A. Organic electronics for neuromorphic computing. Nat. Electron. 1, 386-397 (2018).

46. Yang, J. T. et al. Artificial synapses emulated by an electrolyte-gated tungstenoxide transistor. Adv. Mater. 30, 1801548 (2018).

47. Xu, D. D. et al. Hydrogenation dynamics of electrically controlled metal-insulator transition in proton-gated transparent and flexible $\mathrm{WO}_{3}$ transistors. Adv. Funct. Mater. 29, 1902497 (2019).

48. Deng, X. et al. Proton-mediated phase control in flexible and transparent Mott transistors. Adv. Electron. Mater. 6, 1900742 (2020).

49. Ge, C. et al. Gating-induced reversible $\mathrm{H}_{\mathrm{x}} \mathrm{VO}_{2}$ phase transformations for neuromorphic computing. Nano Energy 67, 104268 (2020).

\section{Acknowledgements}

This work was supported by the Samsung Research Funding Center of Samsung Electronics under Project Number SRFC-MA1401-51.

\section{Author contributions}

K.T.N. and J.-Y.K. conceived the concept and supervised this research. M.-K.S, S.D.N., D.C., and M.J. fabricated the samples and collected the data. M.-K.S., H.S., and T.S. conducted the impedance analysis. H.K. and Y.H.L. performed the material characterization. M.-K.S., Y.-S.L., K.T.N., and J.-Y.K. discussed the results and wrote the manuscript. All authors contributed to the analysis of the data and the final editing of the manuscript.

\section{Competing interests}

The authors declare no competing interests.

\section{Additional information}

Supplementary information is available for this paper at https://doi.org/10.1038/s41467020-19750-5.

Correspondence and requests for materials should be addressed to K.T.N. or J.-Y.K.

Peer review information Nature Communications thanks Themis Prodromakis and the other, anonymous, reviewer(s) for their contribution to the peer review of this work.

Reprints and permission information is available at http://www.nature.com/reprints

Publisher's note Springer Nature remains neutral with regard to jurisdictional claims in published maps and institutional affiliations.

(c) (i) Open Access This article is licensed under a Creative Commons Attribution 4.0 International License, which permits use, sharing, adaptation, distribution and reproduction in any medium or format, as long as you give appropriate credit to the original author(s) and the source, provide a link to the Creative Commons license, and indicate if changes were made. The images or other third party material in this article are included in the article's Creative Commons license, unless indicated otherwise in a credit line to the material. If material is not included in the article's Creative Commons license and your intended use is not permitted by statutory regulation or exceeds the permitted use, you will need to obtain permission directly from the copyright holder. To view a copy of this license, visit http://creativecommons.org/ licenses/by/4.0/.

(C) The Author(s) 2020 